\title{
Rolling Bearings Fault Diagnosis under Variable Conditions Using RCMFE and Improved Support Vector Machine
}

\author{
Xin Zhang and Jian-min Zhao \\ Army Engineering University, Shijiazhuang 050003, PR China.
}

\author{
Hai-ping Li \\ Institute of Systems Engineering Academy of Military Sciences, Beijing 100141, PR China.
}

\author{
Rui-feng Yang \\ Institute of Logistic Science and Technology, Beijing 100071, PR China.
}

\author{
Hong-zhi Teng \\ Unit 32180, Beijing 100039, PR China.
}

(Received 19 January 2019; accepted 2 August 2019)

\begin{abstract}
As critical components, rolling bearings are widely used in a variety of rotating machinery. It is necessary to develop a suitable fault diagnosis method to prevent malfunctions and breakages of bearings during operation. However, the current methods for the fault diagnosis of rolling bearings are too cumbersome to be applied in practical engineering. In addition, the working condition of rolling bearings is generally tough, complex, and especially variable. These conditions cause fault diagnosis methods to be less effective. This paper aims to provide a simple and effective method for the fault diagnosis of rolling bearings under variable conditions. The main contribution of this paper is as follows: (1) The refined composite multiscale fuzzy entropy (RCMFE) is applied in bearing fault feature extraction because of its simplicity and high efficiency; (2) The improved support vector machine (ISVM), based on the whale optimization algorithm (WOA), is proposed to identify the fault pattern of rolling bearings. The ISVM is proposed in this paper to solve the problem that parameter setting affects the classification effect of SVM. In the ISVM, the WOA is employed to optimize both the regularization and kernel parameters of the SVM. Compared with the traditional optimization methods, the WOA has the advantages of high optimization speed and better optimization ability; (3) Combining the RCMFE and the ISVM to diagnose bearing fault under variable working conditions. The effectiveness of the RCMFE-ISVM has been validated via experimental vibration signal of bearings faults under variable working conditions.
\end{abstract}

\section{INTRODUCTION}

Rolling bearings are widely used in various industrial machines such as electric motors, pumps, gearboxes, and turbines. The faults of bearings are prone to occur spalls or cracks on surfaces due to their complex working conditions such as high speed, heavy load, and high temperature. The fault probability of rolling bearings is $30 \%$ in all faults of rotating elements. ${ }^{1}$ It is necessary to detect where the faults occurred in bearings because the faults will lead to both equipment shutdown and safety accidents.

Various methods have been introduced in bearing fault diagnosis, such as vibration analysis, ${ }^{2,3}$ acoustic emission, ${ }^{4-6}$ debris analysis, ${ }^{7,8}$ and infrared thermal imaging. ${ }^{9-11}$ Among those techniques, vibration analysis has served as an efficient tool for bearing fault detection. Various vibration analysis methods have been applied to bearing fault diagnosis, such as autoregressive model, ${ }^{12,13}$ spectral kurtosis, ${ }^{14-16}$ and kurtogram, ${ }^{17}$ wavelet transform, ${ }^{18,19}$ matching pursuit order tracking, ${ }^{20,21}$ and empirical mode decomposition. ${ }^{22,23}$ However, the above methods are complex and require a lot of specific knowledge for their practical application. Moreover, the non- stationary, non-linear characteristics of the bearing fault signal and the complex and diverse working conditions will make fault diagnosis more difficult. Therefore, an intelligent fault diagnosis method for bearings that is based on refined composite multiscale fuzzy entropy (RCMFE) and improved support vector machine (ISVM) is proposed in this paper.

An important and difficult step in rolling bearing fault diagnosis research is fault feature extraction, which directly affects the effect of fault diagnosis. Time domain analysis and frequency analysis are commonly used feature extraction methods, which can extract statistical feature parameters such as energy, root mean square, ${ }^{24}$ etc. These methods are widely used in bearing fault diagnosis because of their simplicity and practicability. However, time and frequency domain analysis can only be applied to stationary and linear signals. To extract fault features from non-stationary and non-linear signals, many methods for time-frequency analysis must be introduced into bearing fault diagnosis. For example, wavelet transform (WT) is generally effective to extract the fault feature of the rolling bearing. ${ }^{25}$ However, the certain bands of the defect information and the selection of the base function are the short- 
comings of WT. Then some self-adaptive time-frequency are applied to the fault diagnosis of the rolling bearing. For instance, empirical mode decomposition (EMD), ${ }^{26}$ local mean decomposition (LMD), ${ }^{27}$ and ensemble empirical mode decomposition (EEMD) ${ }^{28}$ are introduced for feature extraction of the rolling bearing. The problem of mode aliasing has always puzzled the application of EMD. Although LMD and EEMD can alleviate this shortcoming, they have not been completely overcome. To address this shortcoming, Konstantin proposed a signal processing method called variational mode decomposition (VMD) ${ }^{29}$ VMD was quickly introduced into bearing fault feature extraction and produced good results. VMD is introduced into bearing fault feature extraction and there is no obvious mode aliasing, ${ }^{30}$ but VMD needs to set the mode number artificially. Whether the mode number is appropriate or not will affect the effect of fault feature extraction. In addition, the feature extraction process of the above methods is mostly complex, which limits their application in engineering practice. Therefore, it is necessary to explore a simple and effective feature extraction method in engineering practice. Recently, the nonlinear dynamic analysis method has been widely used in bearing fault feature extraction due to its simple and effective characteristics, such as fractal, ${ }^{31}$ sample entropy, ${ }^{32}$ and fuzzy entropy. ${ }^{33}$ However, these methods are all single scale analysis methods, which cannot describe the complex characteristics of vibration signals. Therefore, multi-scale analysis methods are introduced into bearing fault diagnosis, such as multi-scale sample entropy ${ }^{34}$ and multi-scale fuzzy entropy. ${ }^{35}$ Li et al. summarized the application effect of the existing entropy method in the field of rotating machinery fault diagnosis. ${ }^{36,37}$ Because sample entropy uses step function to calculate similarity measure function, there will be a sudden change in measuring similarity. Sample entropy is greatly affected by the sample length. In addition, the MSE and MFE have shortcomings in coarsening. In order to alleviate these problems, Azami proposed a new irregular index called RCMFE and applied it to biomedical signal analysis. ${ }^{38}$ The RCMFE can extract the complexity characteristic related with fault information from vibration signals of rolling bearing, and the RCMFE is quickly applied to bearing fault diagnosis and achieved good results. ${ }^{39,40}$ In literature, the RCMFE needs dimension reduction to be used as feature parameters. ${ }^{37,38}$ The dimension reduction process may be cumbersome and time-consuming, which is not conducive to practical engineering application. In this paper, RCMFE is directly extracted as a feature parameter, and the process of dimension reduction is omitted.

After fault features extraction, it is necessary to classify fault features accurately. The commonly used classification methods mainly include the naive bayesian network (NBN), ${ }^{41}$ the $\mathrm{K}$-nearest neighbor algorithm (KNN), ${ }^{42}$ and the artificial neural network (ANN). ${ }^{43}$ However, each of these methods have their own shortcomings. For instance, the NBN cannot deal with the change results based on feature combination. The KNN calls for a large amount of calculation in classifying test samples. So far, there is no unified and complete theoretical guidance for the selection of the ANN structure, and it can only be selected by experience. Over-selection of network structure, inefficiency in training and over-fitting may result in low network performance and low fault tolerance. If the selection is too small, the network may not converge. Therefore, how to choose the appropriate network structure in application is an important problem. ${ }^{44}$ The support vector machine (SVM) can solve the problem of small sample classification, and it can overcome shortcomings of overfitting and local optimal solution based on structural risk minimization principle. ${ }^{45}$ Therefore, the SVM is applied to early fault detection of bearings. ${ }^{46}$ However, the setting of the parameters will affect the classification effect of SVM. Therefore, several optimization methods are used to optimize the SVM parameters, such as genetic algorithm (GA), ${ }^{47}$ particle swarm optimization (PSO), artificial bee colony algorithm (ABC), ${ }^{48}$ and so on. But the above optimization algorithms all have their shortcomings. For example, the inappropriate choice of operators and parameters used in the evolutionary process makes the GA susceptible to premature convergence. Moreover, the algorithm has other shortcomings, such as time-consuming computation, difficulty in dealing with high-dimensional problems, and so on. ${ }^{49}$ The PSO algorithm is simple in calculation and fast in convergence, but it is easy to fall into local optimum. ${ }^{50}$ The performance of the ABC is better than other methods, but there are still some shortcomings of unbalanced search ability and inadequate resolution accuracy. ${ }^{51}$ The WOA is a novel nature-inspired metaheuristic optimization algorithm proposed by Mirjalili, ${ }^{52}$ and it has been proved that the performance of the method is more competitive than that of the traditional optimization methods. Therefore, this paper proposed an improved SVM which employed the WOA to optimize the parameters of the SVM.

It can be seen from above discussion, there are some problems in fault diagnosis of bearings with variable conditions. Those include:

1. The bearing fault data under variable working conditions have the characteristics of non-linearity and nonstationarity, and the changing working conditions may cause similar characteristics to the fault, which increases the difficulty of fault diagnosis.

2. The method needs to be simplified to facilitate the practical application of engineering.

To cope with the challenges, a novel method based on RCMFE and ISVM is proposed for fault diagnosis of rolling bearing under variable working conditions. This method can automatically identify fault patterns and complete diagnosis with small sample data. The flowchart of the proposed method is shown in Fig. 1. Firstly, the raw vibration signal is collected from rolling bearing fault test rig by sensors, then the RCMFE is extracted as fault features for fault diagnosis. Subsequently, the ISVM is trained by using training data and obtain the ISVM optimal model. Finally, the result of fault identification is obtained by the testing data.

The rest of paper is organized as follows. In Section 2, the refined composite multiscale fuzzy entropy (RCMFE) is introduced. Section 3 describes the improved support vector machine (ISVM) based on whale optimization algorithm (WOA) proposed in this paper. Section 4 and Section 5 investigate the effectiveness of the proposed method by using experimental data. The conclusions are drawn in Section 6. 


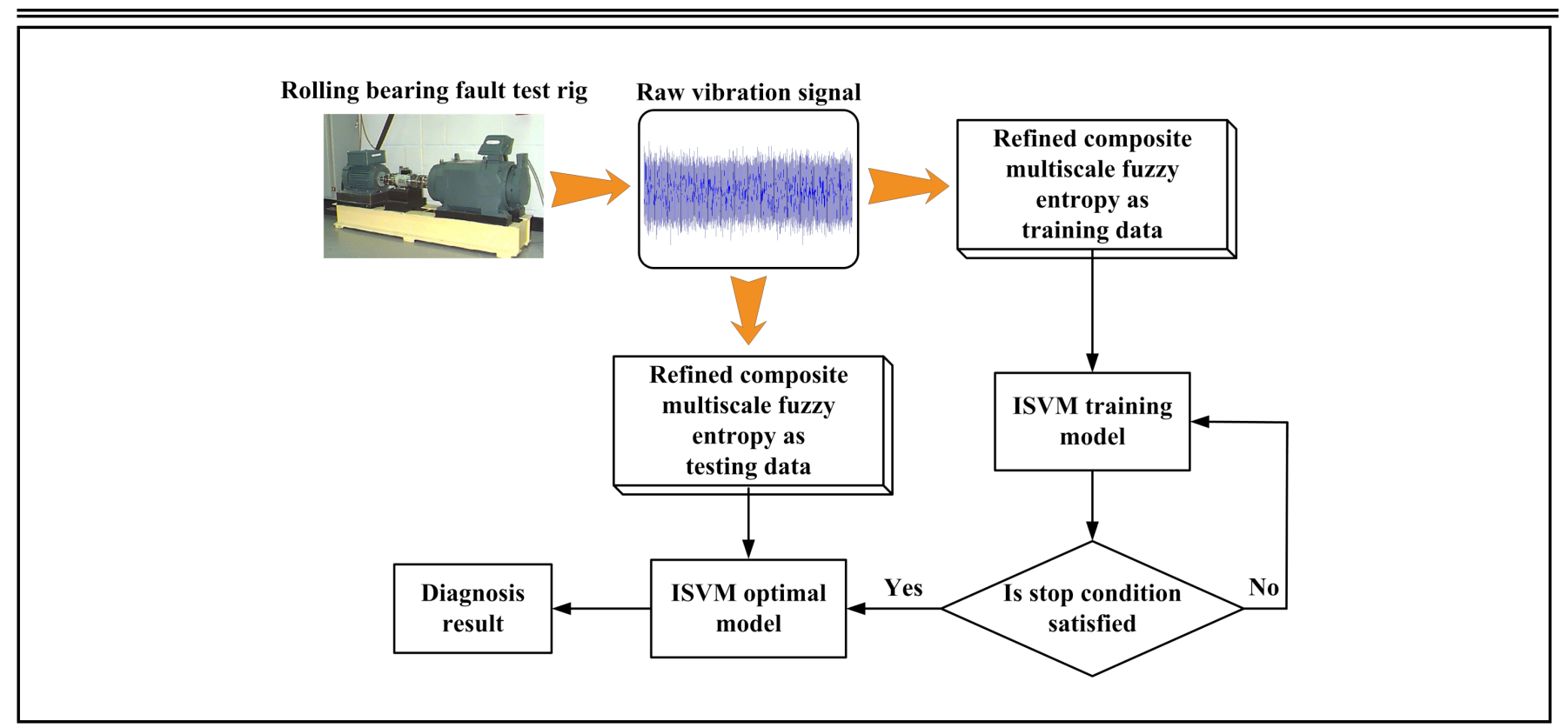

Figure 1. The flow chart of proposed method.

\section{FAULT FEATURE EXTRACTION USING REFINED COMPOSITE MULTISCALE FUZZY ENTROPY}

\subsection{Refined Composite Multiscale Fuzzy Entropy}

Firstly, the concept of fuzzy entropy is introduced in this section. For the time series $\mathbf{y}=\left\{y_{1}, y_{2}, \ldots, y_{N}\right\}, m$ dimensional vectors were obtained as follows: ${ }^{36}$

$$
U_{t}^{m}=\left\{y_{t}, y_{t+1}, \ldots, y_{t+m-1}\right\}-\bar{y}_{t}
$$

where the $\bar{y}_{t}=\frac{1}{m} \sum_{j=0}^{m-1} y_{t+j}$. The distance between $U_{t_{1}}^{m}$ and $U_{t_{2}}^{m}$ was defined as the maximum difference between the corresponding elements, which was calculated as follows:

$$
\begin{aligned}
d_{t_{1} t_{2}} & =d\left[U_{t_{1}}^{m}, U_{t_{2}}^{m}\right] \\
& =\max \left\{\left|U_{t_{1}+k}^{m}-U_{t_{2}+k}^{m}\right|: 0 \leq k \leq m-1, \quad t_{1} \neq t_{2}\right\} .
\end{aligned}
$$

The similarity between $U_{t_{1}}^{m}$ and $U_{t_{2}}^{m}$ was defined by using the fuzzy function, which was shown as follows:

$$
\mu\left(d_{i j}^{m}, n, r\right)=\exp \left(-\left(d_{t_{1} t_{2}}\right)^{n} / r\right)
$$

where $n$ was the power of fuzzy entropy and $r$ was the tolerance. Then the function $\phi^{m}$ was defined as follows:

$$
\begin{aligned}
& \phi^{m}(y, n, r)= \\
& \frac{1}{N-m} \sum_{t_{1}=1}^{N-m} \frac{1}{N-m-1} \sum_{t_{2}=1, t_{1} \neq t_{2}}^{N-m} \exp \left(-\left(d_{t_{1} t_{2}}\right)^{n} / r\right) .
\end{aligned}
$$

Similarly, for dimension $m+1$, repeat the above computation and obtain the $\phi^{m+1}$. The fuzzy entropy was defined as follows:

$$
\operatorname{FuzEn}(y, m, n, r)=\lim _{N \rightarrow \infty}\left(\ln \phi^{m}-\ln \phi^{m+1}\right) .
$$

When $N$ was a finite number, the above expression was expressed as follows:

$$
\operatorname{FuzEn}(y, m, n, r)=\ln \phi^{m}-\ln \phi^{m+1}=-\ln \left(\phi^{m+1} / \phi^{m}\right) .
$$

Then the "coarse-graining" process was applied to the original time series. For the original time series $\left\{x_{1}, x_{2}, \ldots, x_{b}, \ldots, x_{C}\right\}$ whose length is $C$, the embedding dimension $m$ and similarity tolerance $r$ are given in advance, and a new coarse-grained vector was established based on the original time series. And the standard deviation $\sigma$ was proposed as measurement in the coarse-graining process

$$
\begin{gathered}
{ }^{\sigma} y_{i}{ }^{(\tau)}=\sqrt{\frac{1}{\tau} \sum_{b=(i-1) \tau+1}^{i \tau}\left(x_{b}-\frac{1}{\tau} \sum_{b=(i-1) \tau+1}^{i \tau} x_{b}\right)}, \\
1 \leq i \leq\left[\frac{C}{\tau}\right]=N ;
\end{gathered}
$$

where the $\tau$ was time scale factor. Therefore, the $u$-th coarsegrained time series $z_{u}^{\tau}=\left\{{ }^{\sigma} y_{u, 1}{ }^{\tau},{ }^{\sigma} y_{u, 2}{ }^{\tau}, \ldots,{ }^{\sigma} y_{u, p}{ }^{\tau}\right\}$ was generated and it is shown as follows:

$$
{ }^{\sigma} y_{u, j}{ }^{\tau}=\sqrt{\frac{1}{\tau} \sum_{b=u+\tau(j-1)}^{u+\tau j-1}\left(x_{b}-\frac{1}{\tau} \sum_{b=u+\tau(j-1)}^{u+\tau j-1} x_{b}\right)} .
$$

The different time series $z_{u}^{(\tau)},(u=1,2, \ldots, \tau)$ were obtained for different scale factor. The $\phi_{\tau, k}{ }^{m},(k=1,2, \ldots, \tau)$ and $\phi_{\tau, k}{ }^{m+1},(k=1,2, \ldots, \tau)$ are computed separately for a deterministic scale factor $\tau$ and embedding dimension $m$. Finally, the refined composite multiscale fuzzy entropy was obtained as follows:

$$
R C M F E(x, \tau, m, n, r)=-\ln \left(\bar{\phi}_{\tau}^{m+1} / \bar{\phi}_{\tau}^{m}\right)
$$

where the $\bar{\phi}_{\tau}{ }^{m+1}$ and $\bar{\phi}_{\tau}{ }^{m}$ were the mean values of $\phi_{\tau, k}{ }^{m+1}$ and $\phi_{\tau, k}{ }^{m}$ on $1 \leq k \leq \tau$ respectively. 


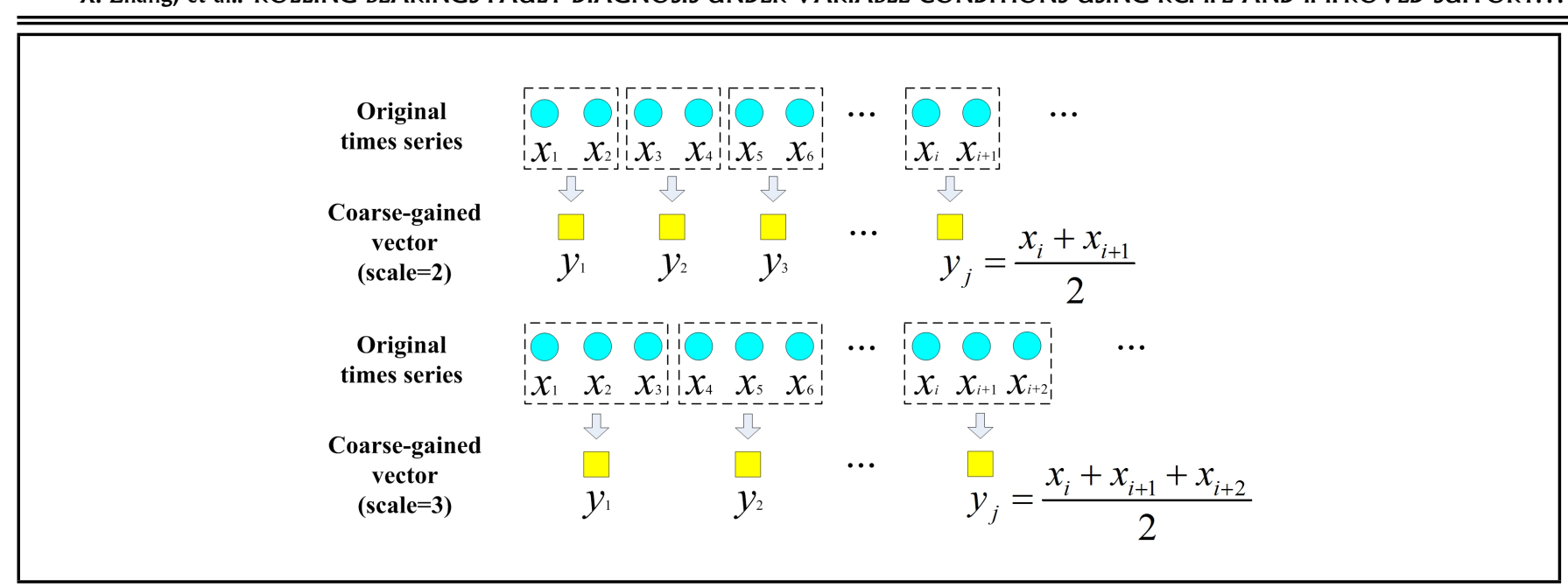

Figure 2. Schematic illustration of the coarse-graining procedure.

The "coarse-graining" process is a concept introduced by Costa to describe the basis and implementation of multiscale entropy method. ${ }^{53}$ Given a one dimensional discrete time series, $\left\{x_{1}, x_{2}, \ldots, x_{b}, \ldots, x_{C}\right\}$, a coarse-grained vector corresponding to the scale factor $\tau$ was constructed as follows. The original times series are divided into nonoverlapping windows of length $\tau$ firstly. Then average the data points inside each window. The "coarse-graining" process is illustrated as Fig. 2. The coarse-grained vector corresponding to the scale factor $\tau$ can be calculated as follows:

$$
y_{j}^{\tau}=\frac{1}{\tau} \sum_{i=(j-1) \tau+1}^{j \tau} x_{i}, \quad 1 \leq j \leq C / \tau .
$$

\subsection{Parameters Selection of RCMFE}

According to the description of RCMFE in the previous section, RCMFE has some parameters that need to be set, such as time delay, similarity tolerance and embedding dimension. Therefore, to make the RCMFE better reflect bearing fault characteristics, it is necessary to select appropriate parameters of RCMFE. In this paper, Euclidean distance, and standard deviation were used to analyze the influence of parameters on RCMFE. Euclidean distance was used to measure the influence of parameters on RCMFE's ability to reflect faults, and standard deviation is used to measure the influence of parameters on RCMFE's stability at different scales.

In this section, an evaluation index based on Euclidean distance was proposed to evaluate RCMFE's ability to reflect fault characteristics. The evaluation index was calculated as follows:

$$
\begin{gathered}
F C I D=\sum_{k=1}^{N}\left[\sum_{i=1}^{M} \sum_{j=1}^{M}\left(R C M F E_{i k}-R C M F E_{j k}\right) / 2\right] \\
i \neq j ;
\end{gathered}
$$

where the $N$ was scale number, and the $M$ was the number of fault states. The FCID was standardized to ranges 0 to 1 . The larger the FCID, the stronger the ability of reflect faults for $R C M F E$, and vice versa. The smaller the standard deviation, the better the stability of $R C M F E$, and vice versa. Therefore, the selection criterion of $R C M F E$ parameters was to make the $F C I D$ as large as possible and the standard deviation as small as possible.

\section{THE IMPROVED SUPPORT VECTOR MACHINE BASED ON WHALE OPTIMIZATION ALGORITHM}

\subsection{Description of Whale Optimization Algorithm}

The WOA is a novel meta-heuristic optimization algorithm that is inspired by the bubble-net hunting strategy for humpback whale. The optimization method consists of three stages: encircling prey, bubble-net attacking method and search for prey.

\subsubsection{Encircling Prey}

Humpback whales can identify their prey and encircled them. It was assumed that the current optimal whale group's individual position (candidate solution) was the target prey location (target prey or close to the best target prey), and its position updating mathematical expression was shown as follows:

$$
\begin{aligned}
\mathbf{X}_{j+1} & =\mathbf{X}_{j}-\mathbf{A} \times \mathbf{D} \\
\mathbf{D} & =\left|\mathbf{C} \times \mathbf{X}_{j}^{*}-\mathbf{X}_{j}\right| ;
\end{aligned}
$$

where $\mathbf{A}$ and $\mathbf{C}$ were coefficient vectors, $\mathbf{X} *$ was the position vector of the best solution obtained so far, $\mathbf{X}$ was the position vector, and the $j$ was the current iteration.

The coefficient vectors $\mathbf{A}$ and $\mathbf{C}$ were computed as follows:

$$
\begin{aligned}
& \mathbf{A}=2 \mathbf{a} \times \mathbf{r}-\mathbf{a} ; \\
& \mathbf{C}=2 \mathbf{r} ;
\end{aligned}
$$

where a was linearly decreased from 2 to 0 over the course of iterations and $\mathbf{r}$ was a random vector in $[0,1]$.

\subsubsection{Bubble-net Attacking Method}

Based on the foraging behaviour of the humpback whale, the mathematical model was established as follows:

1. Shrinking encircling mechanism: this behaviour was achieved by reducing the value of $\mathbf{a}$ in the Eq. (14). It should be noted that the range of variation of $\mathbf{A}$ also shrinks with the decrease of $\mathbf{a}$. The set of random numbers $\mathbf{A}$ is between $[-1,1]$, and the search position of the new whale group can be defined at any position between 
the current whale group's individual location and the best whale group's individual location.

2. Spiral updating position: The mechanism first calculated the distance between the whale group and the prey, and then created a spiral mathematical model between the whale group and the location of its prey to mimic the spiral movement of the humpback whale as follows:

$$
\mathbf{X}_{j+1}=\mathbf{D}^{\prime} e^{b l} \cos (2 \pi l)+\mathbf{X}_{j}^{*}
$$

where $\mathbf{D}^{\prime}=\mathbf{X}_{j}^{*}-\mathbf{X}_{j}$ was the distance between $i$-th whale to the prey, $b$ was a constant for defining the shape of the logarithmic spiral, $l$ was a random number in $[-1,1]$.

The humpback whales swim around the prey within a shrinking circle and along a spiral-shaped path simultaneously. To simulate the synchronization behaviour, it was assumed that a $50 \%$ probability was used as the selection threshold in the process of updating the individual location of the whale group. That is, either the shrinking encircling mechanism was chosen, or the spiral updating position strategy was selected. The mathematical model is shown as follows:

$$
\mathbf{X}_{j+1}=\left\{\begin{array}{ll}
\mathbf{X}_{j}-\mathbf{A} \times \mathbf{D} & p<0.5 \\
\mathbf{D}^{\prime} e^{b l} \cos (2 \pi l)+\mathbf{X}_{j}^{*} & p \geq 0.5
\end{array} ;\right.
$$

where $p$ was a random number in $[0,1]$.

\subsubsection{Search for Prey}

The humpback whales also search for prey randomly. The method based on the variation of $\mathbf{A}$ vector was used to search for prey. The whales can randomly search prey according to their positions. Therefore, $\mathbf{A}$ took a random value greater than 1 or less than -1 , and forced whales to deviate from their prey to search for other more suitable prey to enhance the algorithm's search ability, enabling WOA to perform global search. The mathematical model is shown as follows:

$$
\begin{aligned}
\mathbf{X}_{j+1} & =\mathbf{X}_{\text {rand }}-\mathbf{A} \times \mathbf{D} \\
\mathbf{D} & =\left|\mathbf{C} \times \mathbf{X}_{\text {rand }}-\mathbf{X}_{j}\right| ;
\end{aligned}
$$

where $\mathbf{X}_{\text {rand }}$ was a random position vector chosen from the current population.

\subsection{ISVM Classification Model}

The support vector machine classification model used in this paper was a soft interval nonlinear support vector machine. Given a training data set $D=\left\{\mathbf{x}_{i}, y_{i}\right\}_{i=1}^{n}$, where $\mathbf{x}_{i} \in R^{n}$ was the $i$-th input feature vectors, $y_{i} \in\{+1,-1\}$ was the class label of $\mathbf{x}_{i}, n$ was the number of all samples. Using linear soft interval support vector machines to construct optimal classification hyperplanes should be satisfied:

$$
\left\{\begin{array}{ll}
\mathbf{w} \cdot \mathbf{x}_{i}+b \geq+1-\xi_{i} & y_{i}=+1 \\
\mathbf{w} \cdot \mathbf{x}_{i}+b \geq-1+\xi_{i} & y_{i}=-1
\end{array} .\right.
$$

Two constraint conditions are merged into:

$$
y_{i}\left[\left(\mathbf{w} \cdot \mathbf{x}_{i}\right)+b\right]-1+\xi_{i} \geq 0, \quad i=1,2, \ldots, n ;
$$

where the $\mathrm{w}$ was weight vector, $b$ was a scalar, and the $\xi_{i} \geq 0$ was slack variables. And in the case of nonlinearity, it was necessary to map the input $\mathbf{x}_{i}$ to a new high-dimensional feature set $\varphi\left(\mathbf{x}_{i}\right)$ through a nonlinear mapping $\varphi$. Therefore, the classification constraint was converted to as follows:

$$
y_{i}\left[\left(\mathbf{w} \cdot \boldsymbol{\varphi}\left(\mathbf{x}_{i}\right)\right)+b\right]-1+\xi_{i} \geq 0, \quad i=1,2, \ldots, n .
$$

The objective function was

$$
\min \frac{1}{2} \mathbf{w}^{T} \mathbf{w}+C \sum_{i=1}^{n} \xi_{i}
$$

where $C$ was the regularization parameter, which determined the trade-off between experience risk and complexity. To solve the problem the following Lagrange function was constructed

$$
\begin{aligned}
L(\mathbf{w}, b, \boldsymbol{\xi}, \boldsymbol{\alpha}, \boldsymbol{\beta})= & \frac{1}{2} \mathbf{w}^{T} \mathbf{w}+C \sum_{i=1}^{n} \xi_{i}-\sum_{i=1}^{n} \beta_{i} \xi_{i}- \\
& \sum_{i=1}^{n} \alpha_{i}\left[y_{i}\left(\left(\mathbf{w} \cdot \boldsymbol{\varphi}\left(\mathbf{x}_{i}\right)\right)+b\right)+\xi_{i}-1\right] ;
\end{aligned}
$$

where $\alpha_{i} \geq 0, \beta_{i} \geq 0$ were Lagrange multipliers. The partial derivatives of $\mathbf{w}, b$ and $\xi_{i}$, and they were equal to 0 , which can be obtained as follows:

$$
\begin{aligned}
L(\mathbf{w}, b, \boldsymbol{\xi}, \boldsymbol{\alpha}, \boldsymbol{\beta})= & \sum_{i=1}^{n} \alpha_{i}- \\
& \frac{1}{2} \sum_{i=1}^{n} \sum_{j=1}^{n} \alpha_{i} \alpha_{j} y_{i} y_{j}\left[\varphi\left(x_{i}\right) \cdot \varphi\left(x_{j}\right)\right] .
\end{aligned}
$$

According to the Karush-Kuhn-Tucker (KKT) condition, the solution of the optimization problem must also be satisfied as follows:

$$
\left\{\begin{array}{l}
\alpha_{i}\left[y_{i}\left(\left(\mathbf{w} \cdot \boldsymbol{\varphi}\left(\mathbf{x}_{i}\right)\right)+b\right)+\xi_{i}-1\right]=0 \\
\beta_{i} \xi_{i}=0 \Rightarrow\left(C-\alpha_{i}\right) \xi_{i}=0, \quad i=1,2, \ldots, n
\end{array}\right.
$$

According to the Mercer condition, kernel function $K\left(\mathbf{x}_{i}, \mathbf{x}_{j}\right)$ can replace the inner product in the feature space. Therefore, the soft interval nonlinear support vector machine can be transformed into the following dual two programming problem:

$$
\begin{array}{r}
\max \sum_{i=1}^{n} \alpha_{i}-\frac{1}{2} \sum_{i=1}^{n} \sum_{j=1}^{n} \alpha_{i} \alpha_{j} y_{i} y_{j} K\left(\mathbf{x}_{i}, \mathbf{x}_{j}\right) \\
\text { s.t. }\left\{\begin{array}{l}
\sum_{i=1}^{n} y_{i} \alpha_{i}=0 \\
0 \leq \alpha_{i} \leq C, \quad i=1,2, \ldots, n
\end{array} .\right.
\end{array}
$$

After solving the above problem, the optimal classification function was obtained as follows:

$$
f(\mathbf{x})=\operatorname{sgn}\left[\sum_{i=1}^{n} \alpha_{i} y_{i} K\left(\mathbf{x}_{i}, \mathbf{x}\right)+b\right] .
$$

In this paper, the kernel function of the support vector machine was a radial basis function (RBF) which shown as follows:

$$
K\left(\mathbf{x}_{i}, \mathbf{x}\right)=\exp \left(\gamma\left\|\mathbf{x}_{i}-\mathbf{x}\right\|^{2}\right), \quad \gamma>0 ;
$$




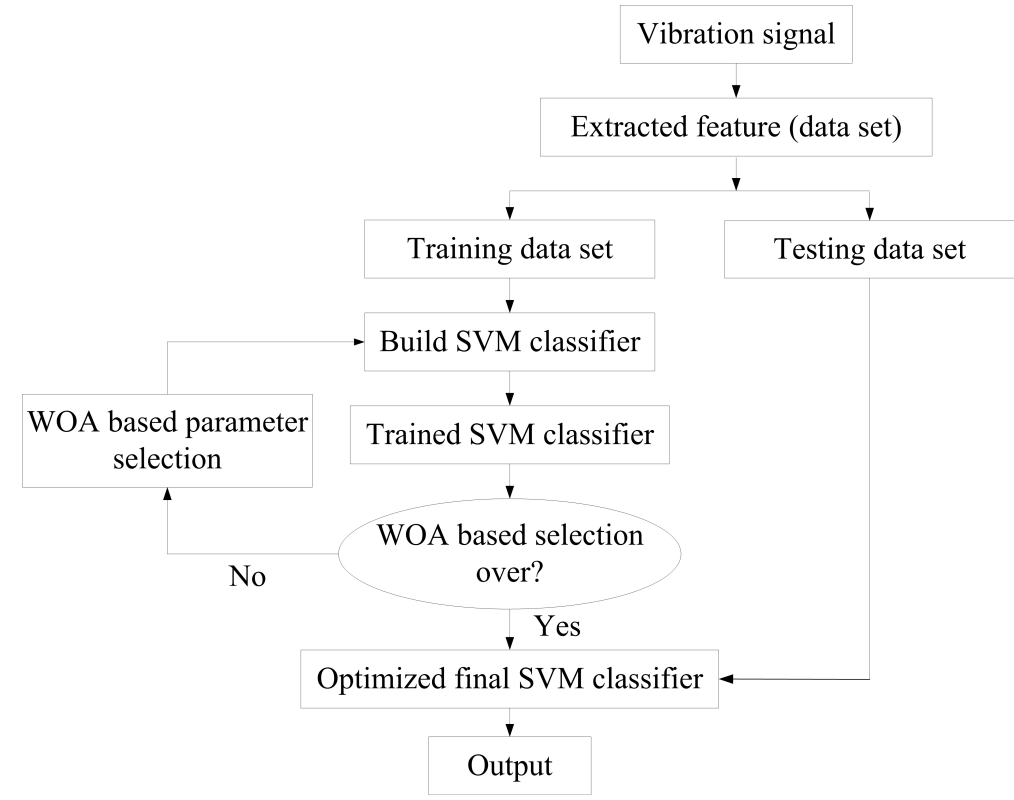

Figure 3. Flow chart for optimization of SVM parameters by WOA.

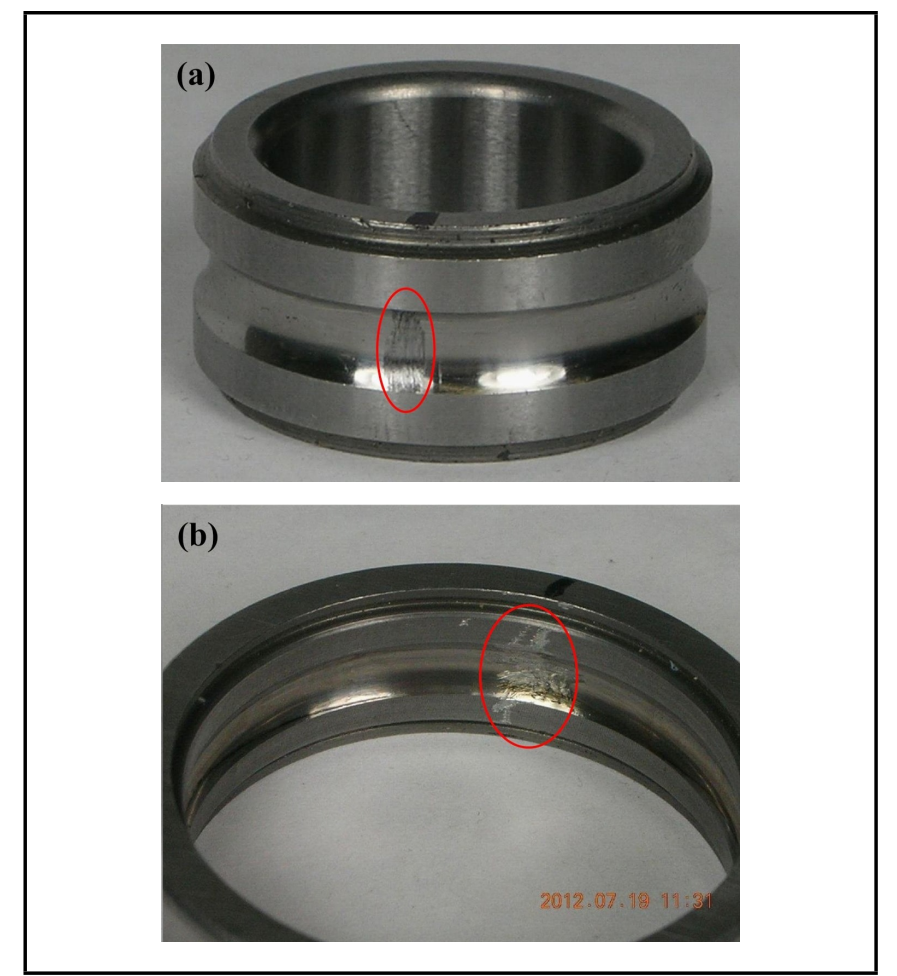

Figure 4. Fault bearing: (a) inner race fault, (b) outer race fault.

where $\gamma$ was the kernel parameter.

Therefore, the regularization parameter $C$ and RBF kernel parameter $\gamma$ are two parameters that can affect the classification efficiency of SVM. In this paper, the WOA is used to optimize these parameters. A fitness function is proposed to evaluate the optimization effect of the algorithm which is shown as follows:

$$
F_{\text {fit }}\left(C_{i}, \gamma_{i}\right)=1-\frac{1}{1+a_{\mathrm{vc}}\left(C_{i}, \gamma_{i}\right)}
$$

where $a_{\mathrm{vc}}\left(C_{i}, \gamma_{i}\right) \in[0,1]$ is the cross validation accuracy of SVM using the parameters $C_{i}$ and $\gamma_{i}$. The flow chart for optimization of SVM parameters by WOA is shown as Fig. 3.

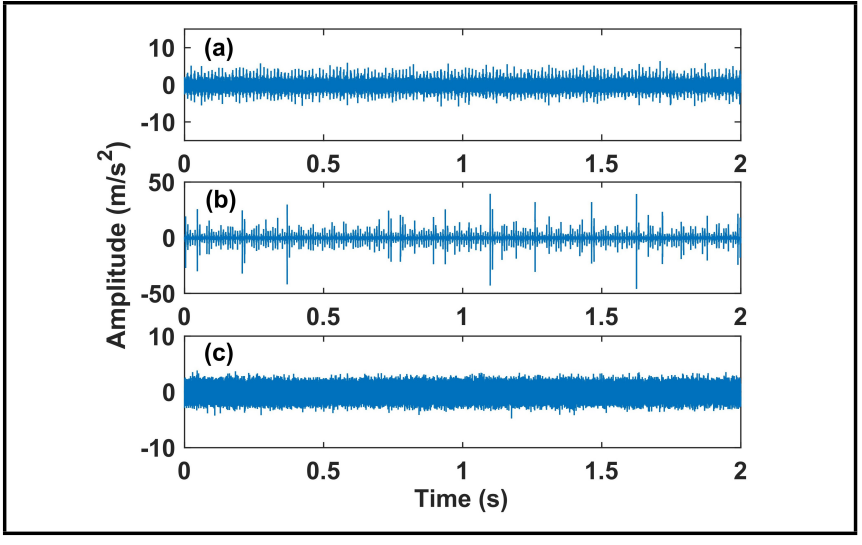

Figure 5. Waveform of raw signals: (a) outer race fault, (b) inner race fault, (c) normal state.

\section{CASE STUDY 1}

In this section, the fault data provided by Society for Machinery Failure Prevention Technology (MFPT) was used to verify the effectiveness of the proposed method. The fault data sets contained the data of outer race fault, inner race fault and normal state. The location of outer race fault and inner race fault are shown in Fig. 4. Figure 5 displays the time domain waveform of raw signals for outer race fault, inner race fault and normal state. The rotation frequency was $25 \mathrm{~Hz}$ in the experiment. There were seven working conditions for the outer race fault data, including 25, 50, 100, 150, 200, 250 and $300 \mathrm{lbs}$ of load. There were seven working conditions for the inner race fault data, including $0,50,100,150,200,250$ and $300 \mathrm{lbs}$ of load. The load of normal state data was $270 \mathrm{lbs}$. The data of the above each mode was divided into 84 samples, a total of 252 samples, half of which were used as train data, and the rest were used as test data.

The influence of parameters on RCMFE was analyzed before applying the proposed method to fault diagnosis. Firstly, the influence of different time delay $d$ on RCMFE was analyzed. The analysis result of different time delay $d$ on RCMFE 


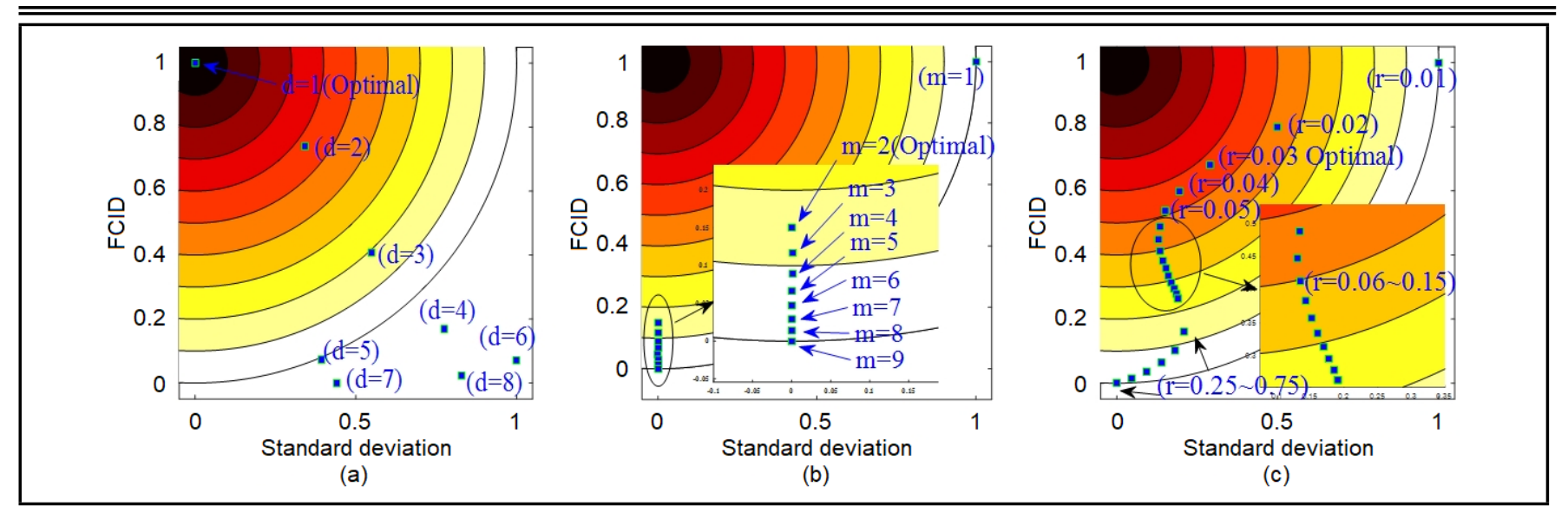

Figure 6. Analysis results of RCMFE parameters: (a) different time delay $d$, (b) different embedding dimension $m$, (c) different similarity tolerance $r$.

Table 1. Input parameters of WOA in ISVM.

\begin{tabular}{|c|c|}
\hline Parameter & Value \\
\hline Number of whales population & 50 \\
Maximum number of iterations & 100 \\
Parameter lower bound & 0.0001 \\
Parameter upper bound & 100 \\
Number of cross validation & 3 \\
\hline
\end{tabular}

was displayed in Fig. 6(a). As shown in Fig. 6(a), the abscissa was standard deviation and the ordinate was FCID. After standardization of FCID and standard deviation, the ranges of values both were 0 to 1 . According to the description of parameter selection in Section 2, when FCID was 1 and standard deviation was 0 , the optimal result was obtained. Therefore, several concentric circles were drawn with $(0,1)$ as the centre of the circle, and scattered points calculated with different parameters fell into the concentric circle. The closer the scatter point was to the centre of the circle, the better the parameter was. As can be seen from Fig. 6(a), when time delay $d$ took 1 , the scattering point fell at the centre of the circle, which showed that the result was the best. So, the time delay is set to 1 . Moreover, it was found that the greater the value of $d$, the worse the ability to reflect fault characteristics and stability of RCMFE.

The influence of embedding dimension $m$ on RCMFE was analyzed in this section. The analysis results for different embedding dimension $m$ on RCMFE are shown in Fig. 6(b). When $m$ equals 1 , the value of FCID reached the maximum, but the standard deviation reached the maximum simultaneously. Therefore, for the sake of stability, $m$ cannot take 1 . The standard deviation was very small and stable when $m$ was in the range of 2 to 9 , and the value of FCID was the largest when $m$ was 2 . Therefore, the embedding dimension $m$ was set to 2 .

The influence of different similarity tolerance $r$ on RCMFE was analyzed in this section. The analysis results of different similarity tolerance $r$ on RCMFE are shown in Fig. 6(c). When $r$ increased gradually from 0.01 , the distribution of scatters first approached the centre of the circle, and then gradually moved away. It can be found from the graph that the scatter point at $r=0.03$ was closest to the centre of the circle. Therefore, the similarity tolerance was set to 0.03 in the case.

After parameters analysis of RCMFE, the proposed method was employed to diagnose the bearing fault data mentioned above. Then the RCMFE of all the samples were extracted as the fault feature of bearings. The feature vector composed of RCMFE was used as the input of the ISVM, and then the

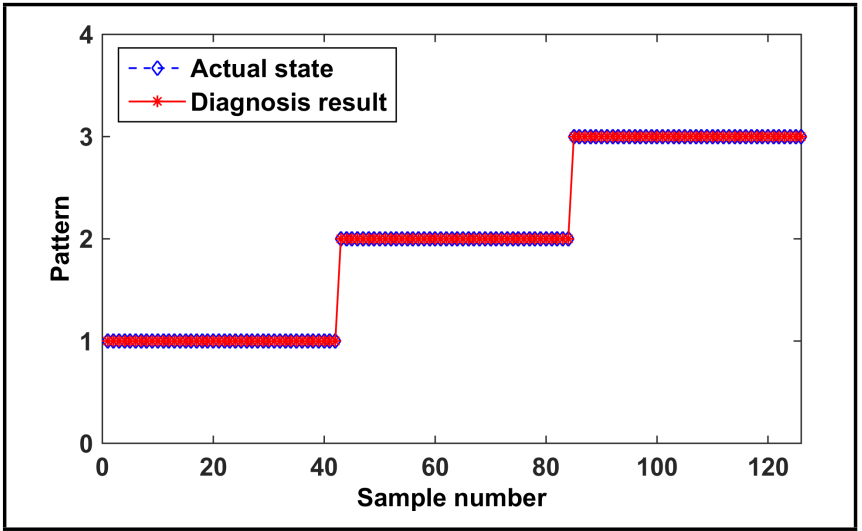

Figure 7. Diagnosis results of proposed method.

bearing fault diagnosis was carried out. The WOA parameter settings in the ISVM are shown in the Table 1. After optimization, the optimized $C$ and $\gamma$ were 6.6872 and 0.0073 , respectively. The diagnosis result is presented in Fig. 7. As shown in the Fig. 7, the fault diagnosis results were in good agreement with the actual state. After calculated, the recognition accuracy of bearing fault pattern was $100 \%$. It is illustrated that the proposed method was effective for fault diagnosis of rolling bearings.

To verify the advantage of RCMFE in fault detection for rolling bearings, two typical and popular feature parameters of multiscale fuzzy entropy (MFE) and multiscale sample entropy (MSE) were selected as the fault features. The parameters of MFE and MSE were consistent with RCMFE. After calculation, the feature vectors of MFE and MSE were used as input for the ISVM, respectively. The diagnosis results of using MFE and MSE as fault feature are presented in Fig. 8. As shown in Fig. 8(a), two samples are misdiagnosed. One inner race fault sample is misdiagnosed as outer race fault and the other outer race fault sample is misdiagnosed as normal state. After calculation, the recognition accuracy of the bearing fault pattern with using MFE-ISVM was $98.4127 \%$. As shown in Fig. 8(b), three samples are misdiagnosed. Two inner race fault samples are misdiagnosed as outer race fault, and the other outer race fault sample is misdiagnosed as inner race fault. The recognition rate of using MSE-ISVM was $97.6190 \%$. Therefore, it is illustrated that the performance of RCMFE in bearing fault diagnosis was more competitive than the performance of MFE and MSE.

To further verify the advantages of the WOA in the ISVM, 


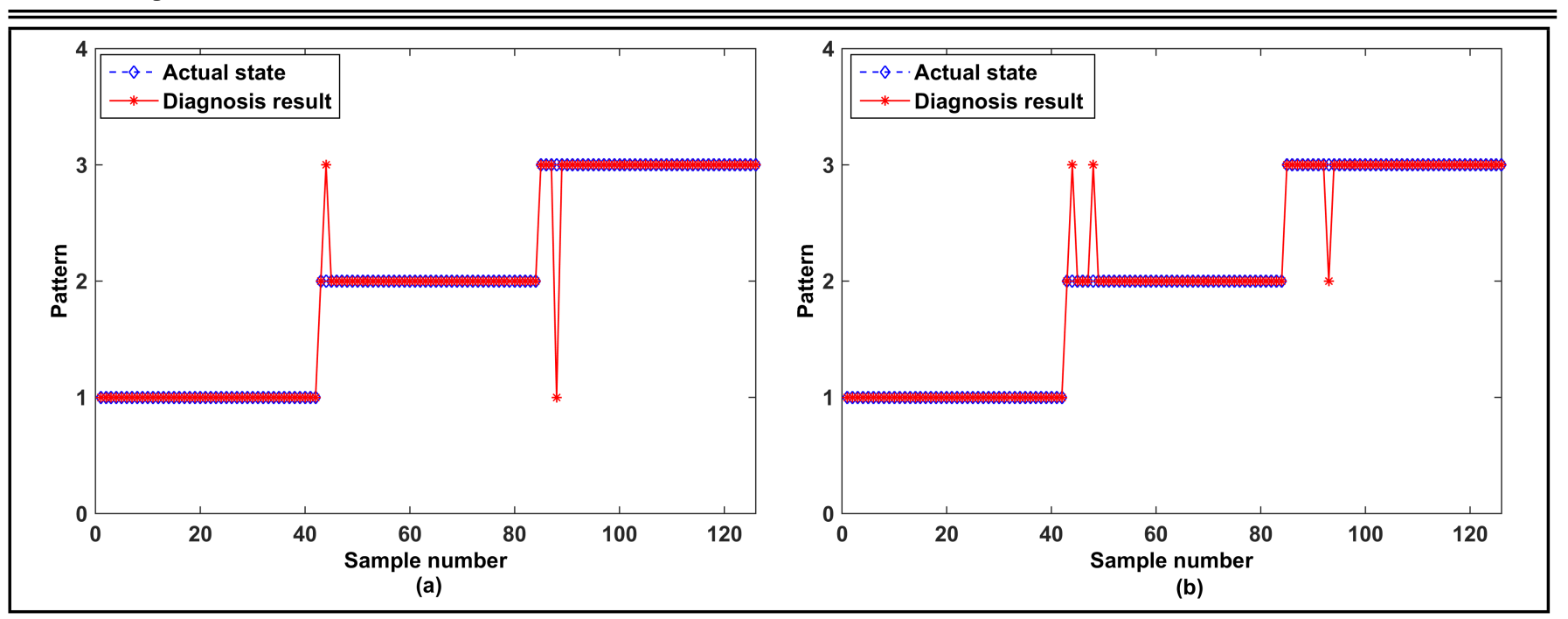

Figure 8. Diagnosis results of MFE-ISVM and MSE-ISVM: (a) MFE-ISVM, (b) MSE-ISVM.

Table 2. Optimization results of methods.

\begin{tabular}{|c|c|c|c|c|c|c|}
\hline Method & Optimal fitness & Recognition rate & Optimal value of $C$ & Optimal value of $\gamma$ & Number of iterations & Runtime \\
\hline WOA-SVM & 0.5 & $100 \%$ & 6.6872 & 0.0073 & 2 & $5.174758 \mathrm{~s}$ \\
GA-SVM & 0.5 & $99.2063 \%$ & 0.4856 & 39.5706 & $8.784553 \mathrm{~s}$ \\
PSO-SVM & 0.5 & $100 \%$ & 30.9112 & 0.0001 & 4 \\
ABC-SVM & 0.4979 & $100 \%$ & 8.3482 & 9.0499 & $6.685555 \mathrm{~s}$ \\
\hline
\end{tabular}

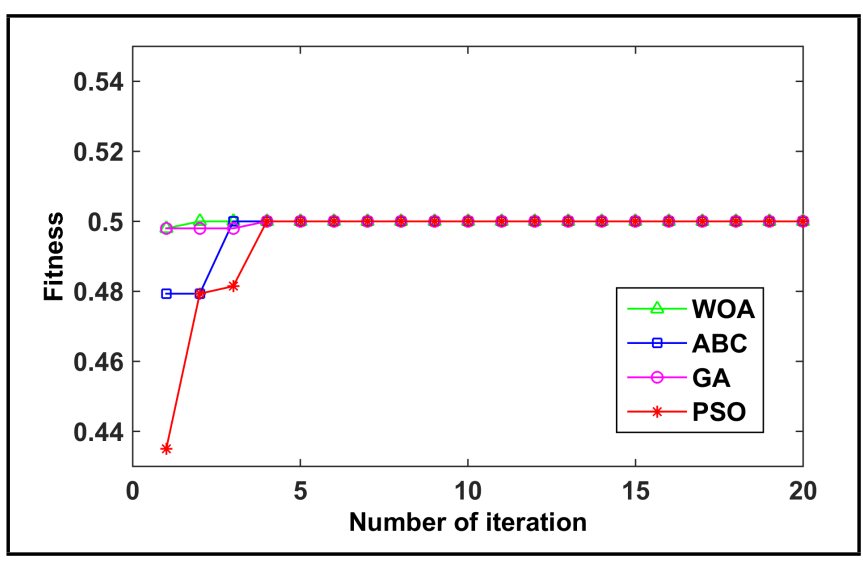

Figure 9. Iterative process of several optimization methods.

three traditional optimization methods of genetic algorithm (GA), particle swarm optimization (PSO) and artificial bee colony algorithm (ABC) were applied to optimize the parameters of the SVM. The input parameters of the three algorithms were consistent with the WOA. The performance of these methods is displayed in Table 2 . The optimization iteration process of these methods is presented in Fig. 9. As shown in Fig. 9, the fitness functions reached maximum and kept unchanged when the four optimization methods iterated to a certain number of times. However, the WOA was faster than the other algorithms in searching for the optimal solution. The optimal solution was found in the second iteration. It shows that the performance of WOA was more competitive than other traditional optimization methods.

To prove the effectiveness of the ISVM, three traditional classification method of naive bayesian network (NBN), artificial neural network (ANN) and K-nearest neighbors (KNN) were employed to classify the bearing faults combined with RCMFE. Figure 10(a) displayed the diagnosis result of using the NBN as the classification method, and there were twelve samples being misdiagnosed by using this method. Three
Table 3. The recognition rate of different classification methods.

\begin{tabular}{|c|c|c|c|}
\hline Method & $\begin{array}{c}\text { Number of correct } \\
\text { identification samples }\end{array}$ & $\begin{array}{c}\text { Number of } \\
\text { test samples }\end{array}$ & $\begin{array}{c}\text { Recognition } \\
\text { rate }\end{array}$ \\
\hline RCMFE-ISVM & 126 & 126 & $100 \%$ \\
RCMFE-NBN & 114 & 126 & $90.48 \%$ \\
RCMFE-ANN & 120 & 126 & $95.24 \%$ \\
RCMFE-KNN & 118 & 126 & $93.65 \%$ \\
\hline
\end{tabular}

of the normal state samples were misdiagnosed as outer race faults, and nine of the outer race fault samples were misdiagnosed as the normal state. The diagnosis result of using ANN as the classification method is presented in Fig. 10(b). There were six samples of outer race fault being misdiagnosed as inner race fault in the Fig. 10(b). The diagnosis result of using the $\mathrm{KNN}$ as a classification method is shown as Fig. 10(c). As shown in Fig. 10(c), eight samples were misdiagnosed. Two samples of normal state were misdiagnosed as outer race fault state, and six samples of outer race fault state were misdiagnosed as normal state. The recognition rates of these methods were presented in Table 3. It can be found from Table 3 that the recognition rates of the ISVM were higher than other traditional classification methods. It is illustrated that the performance of the ISVM was more competitive than other traditional methods.

The influence of different numbers of training samples on diagnostic recognition rate was analyzed. As shown in Fig. 11, with the decrease of training samples, the recognition rate of the ISVM decreased, but all recognition rates remained above $95 \%$. In contrast, with the decrease of training samples, the overall recognition rate of other methods decreased more seriously. It demonstrates that the ISVM proposed in this paper was more robust to training samples than other methods. The ISVM can still maintain a high recognition rate in the case of small samples, which was more suitable for practical application.

In addition, the influence of the different scale factor on diagnostic recognition rate was analyzed in this section. As 


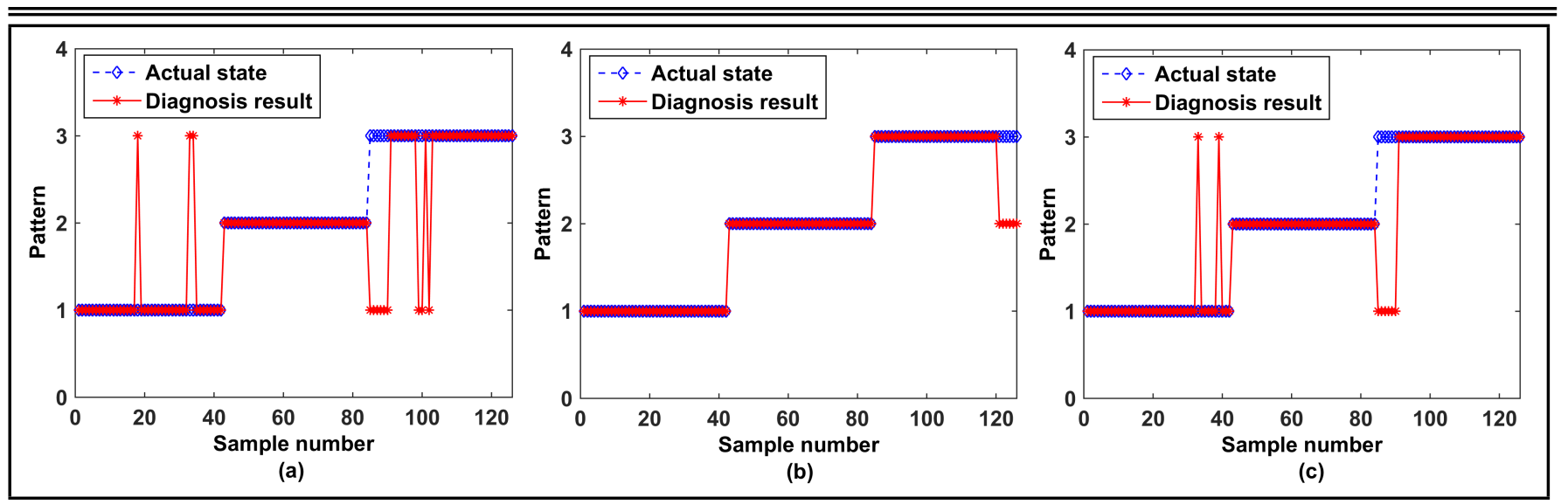

Figure 10. Diagnosis results of using different classification methods: (a) NBN, (b) ANN, (c) KNN.

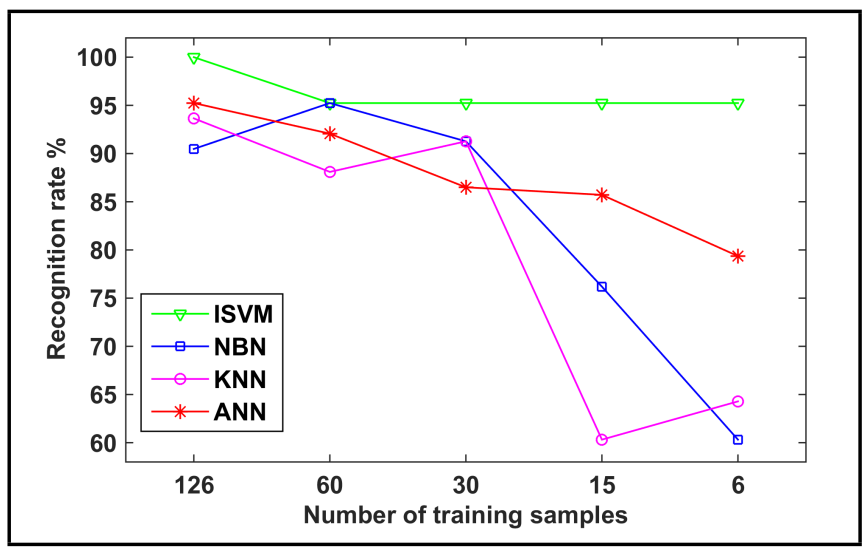

Figure 11. Fault recognition rate of different training samples.

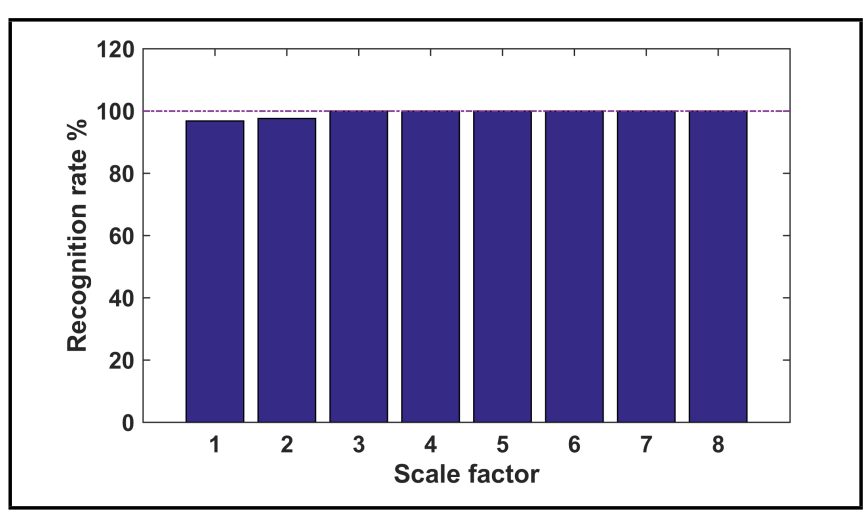

Figure 12. Fault recognition rate of different scale factor.

shown in Fig. 12, the recognition rate increased with the increase of scale factor. When the scale factor was greater than or equal to 3 , the recognition rate reached $100 \%$. It shows that the fault recognition rate reached the maximum value and remained unchanged after the scale factor was greater than 3 . If the scale factor was too large, the amount of calculation increased. Therefore, it was more appropriate to set the scale factor at $3 \sim 6$.

\section{CASE STUDY 2}

In Case 1, three fault patterns of rolling bearings were diagnosed by the proposed method and the results show that the method was effective. However, the data in Case 1 had fewer fault patterns and it was not difficult to diagnose the faults in this case. In this section, the fault data of the Case Western

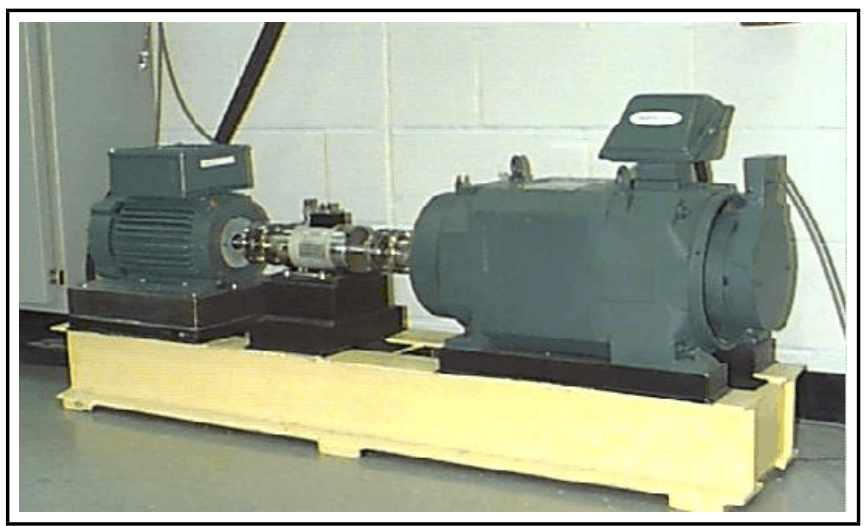

Figure 13. Structure of test rig.

Table 4. Details of different state data.

\begin{tabular}{|c|c|c|c|}
\hline $\begin{array}{l}\text { Pattern } \\
\text { number }\end{array}$ & $\begin{array}{c}\text { Fault level } \\
\text { (inch) }\end{array}$ & $\begin{array}{c}\text { Fault } \\
\text { location }\end{array}$ & Working condition \\
\hline 1 & 0.007 & Rolling element & \\
\hline 2 & 0.007 & Inner race & Working condition 1 : \\
\hline 3 & 0.007 & Outer race & 0 horsepower load, 1797 rpm speed; \\
\hline 4 & 0.014 & Rolling element & Working condition 2 : \\
\hline 5 & 0.014 & Inner race & 1 horsepower load, 1772 rpm speed; \\
\hline 6 & 0.014 & Outer race & Working condition 3 : \\
\hline 7 & 0.021 & Rolling element & 2 horsepower load, 1750 rpm speed; \\
\hline 8 & 0.021 & Inner race & Working condition 4 : \\
\hline 9 & 0.021 & Outer race & 3 horsepower load, $1730 \mathrm{rpm}$ speed. \\
\hline 10 & \multicolumn{2}{|c|}{ Normal state } & \\
\hline
\end{tabular}

Reserve University were also employed to verify the performance of the proposed method in the bearing fault detection, and the test rig is shown in Fig. 13. In the last case, the proposed method was just used to diagnose the data of different fault pattern. In this case, the data with different fault and different fault degrees was used to verify the effectiveness of the proposed method in the bearing fault detection. The data contained signals of three fault modes of bearings, such as outer race fault, inner race fault and rolling element fault. Each fault pattern has three levels of fault: 0.007 inches, 0.014 inches and 0.021 inches. So, there were nine kinds of fault pattern and one kind of normal state data. The data for each state hd four working conditions. The details of the data are shown in Table 4. Figure 14 displays the time domain waveform of ten patterns row signal under working condition 1 . The data of each state was divided into 40 samples, a total of 400 samples, half of which were used as train data, and the rest were used as test data. 


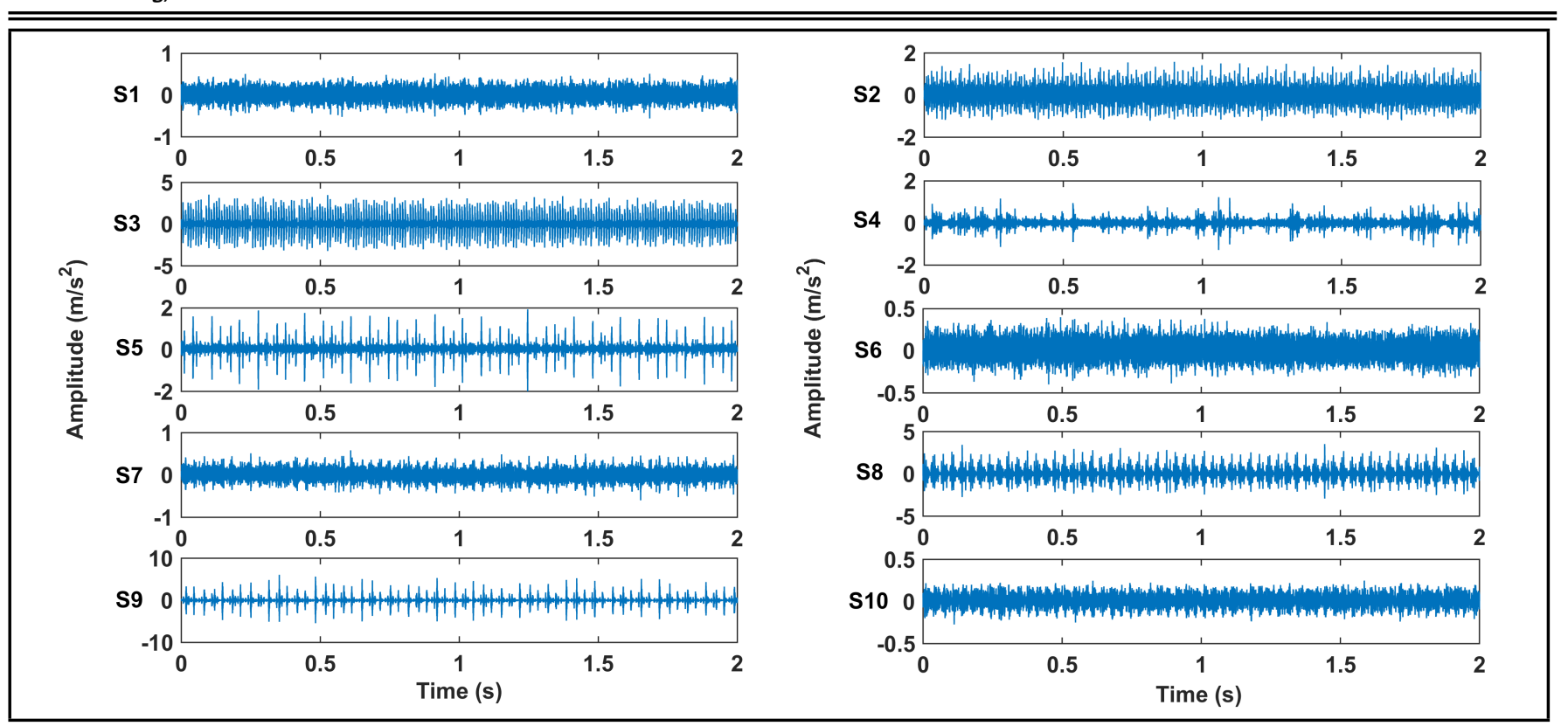

Figure 14. Waveform of raw signals under working condition 1.

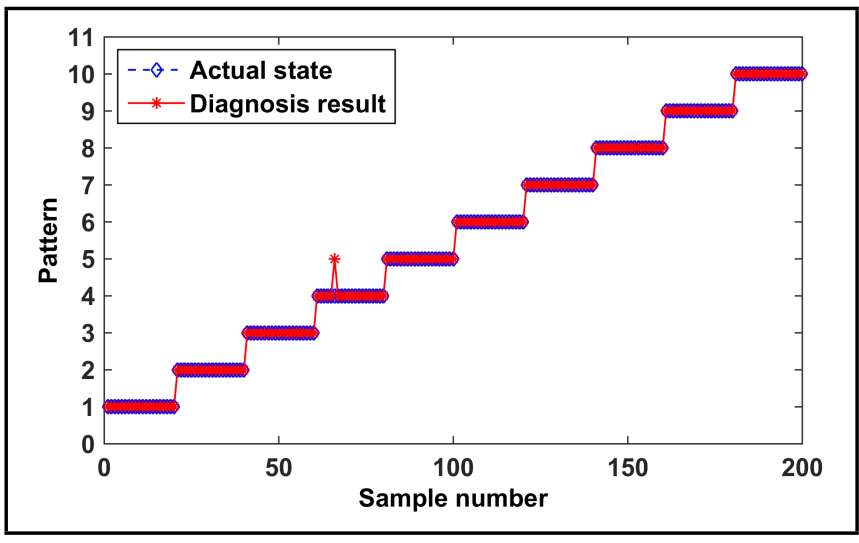

Figure 15. Diagnosis results of proposed method.

The proposed method was employed to diagnose the bearing fault data mentioned above. The fuzzy power and embedding dimension of refined composite multiscale fuzzy entropy were both set to 2 , and the tolerance was 0.03 . The scale factor of refined composite multiscale fuzzy entropy was set to 6 . Then, the feature vector composed of RCMFE was extracted as the input of the ISVM, and the bearing fault diagnosis was carried out. The WOA parameter settings in the ISVM were consistent with Case 1. After optimization, the optimized $C$ and $\gamma$ were 9.3051 and 6.4356 , respectively. The diagnosis result was presented in Fig. 15. As shown in Fig. 15, one sample of fault state 4 was misdiagnosed as fault state 5 . The recognition rate was $99.5 \%$ after calculation, which showed that the proposed method was effective for bearing fault detection.

As in Case 1, MFE and MSE were used as fault features for diagnosis and the diagnosis results are presented in Fig. 16. As shown in Fig. 16(a), there were four samples misdiagnosed. In details, one sample of pattern 1 was misdiagnosed as pattern 7 , two samples of pattern 4 were misdiagnosed as pattern 2 and pattern 7 respectively, one sample of pattern 7 was misdiagnosed as pattern 2. As shown in Fig. 16(b), there were six samples misdiagnosed, in which two samples of pattern 4 and two samples of pattern 7 were misdiagnosed as pattern 2, one sample of pattern 4 was misdiagnosed as pattern 1, one sam- ple of pattern 5 was misdiagnosed as pattern 4. After calculation, the recognition accuracy of bearing fault pattern using the MFE-ISVM and MSE-ISVM was $98 \%$ and $97 \%$ respectively. Therefore, it was illustrated that the performance of the RCMFE in bearing fault diagnosis is more competitive than the performance of the MFE and MSE.

To prove the optimization efficiency of the WOA, the traditional optimization methods such as GA, PSO and ABCA were also applied to optimize the parameters of the SVM. The input parameters of the three optimization methods were the same with those of the WOA. The optimization iteration process of these methods was presented in Fig. 17. As shown in Fig. 17, the iteration times of the WOA, ABC, GA and PSO to reach the optimal fitness function were $2,2,31$ and 6 respectively. The optimal fitness of the WOA is higher than that of other methods. The optimization results and recognition accuracy of the above methods are shown in Table 5. The recognition rates of the WOA-SVM, GA-SVM and PSO-SVM were 99.5\%, while that of the ABC-SVM was only $85.5 \%$. In addition, the run time of the WOA-SVM was shorter than the other methods. It shows that the performance of the WOA was more competitive than other traditional optimization methods.

To prove the effectiveness of the ISVM, the NBN, KNN and ANN were employed as classifiers respectively to detect the bearing. The fault diagnosis results of the above methods are displayed in Fig. 18. As shown in Fig. 18, most of the misdiagnosis samples were concentrated in pattern 4 and pattern 7 . It shows that the RCMFE values had some confusion between the samples of pattern 4 and samples of pattern 7 . The recognition rates of these methods are presented in Table 6 . It can be found from the Table 6 that the recognition rates of the ISVM were higher than other traditional classification methods. It is illustrated that the performance of the ISVM was more competitive than other traditional methods.

Like Case 1, the influence of different numbers of training samples on diagnostic recognition rate was analyzed. As shown in Fig. 19, with the decrease of training samples, the recognition rates of all methods showed a downward trend. But compared with other methods, the ISVM had a gentle down- 


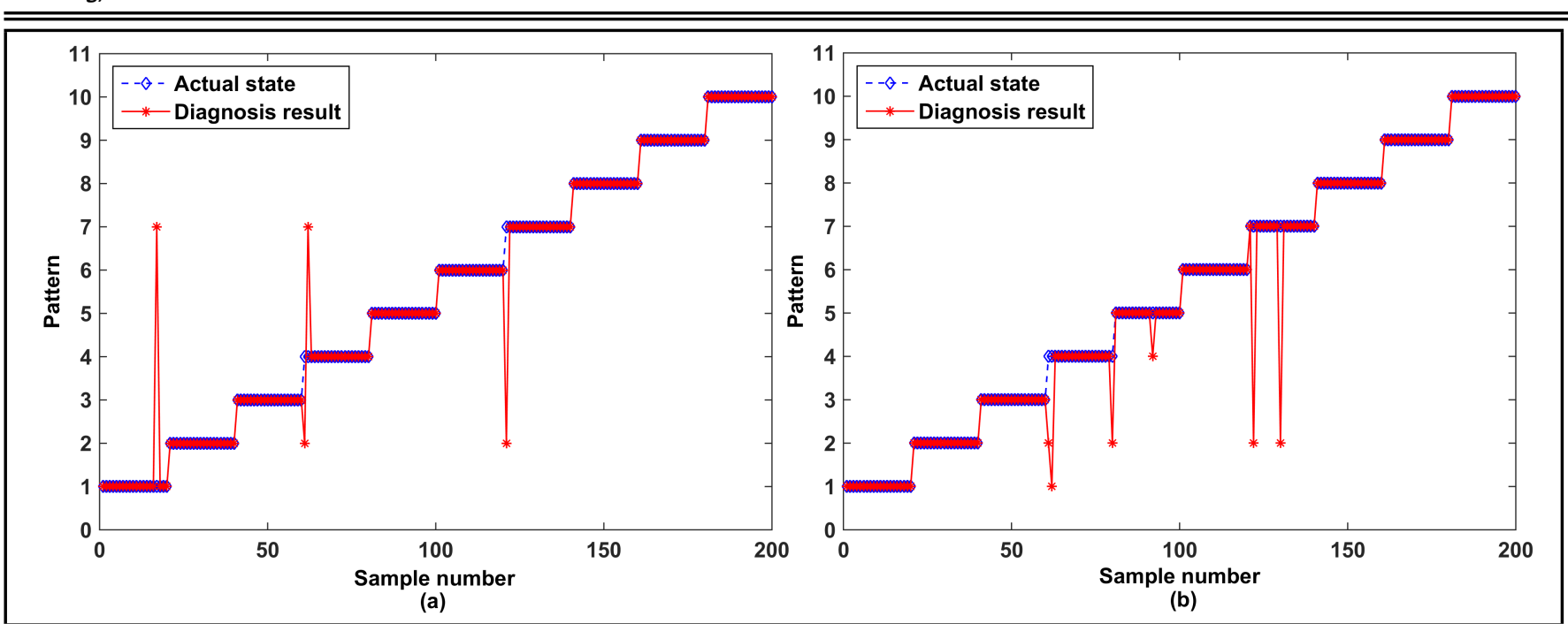

Figure 16. Diagnosis results of MFE-ISVM and MSE-ISVM: (a) MFE-ISVM, (b) MSE-ISVM.

Table 5. Optimization results of methods.

\begin{tabular}{|c|c|c|c|c|c|c|}
\hline Method & Optimal fitness & Recognition rate & Optimal value of $C$ & Optimal value of $\gamma$ & Number of iterations & Runtime \\
\hline WOA-SVM & 0.4937 & $99.5 \%$ & 9.3051 & 6.4356 & 2 & $5.953217 \mathrm{~s}$ \\
GA-SVM & 0.4924 & $99.5 \%$ & 5.0071 & 30.2239 & 31 \\
PSO-SVM & 0.4924 & $99.5 \%$ & 4.490 & 29.6335 & 6 \\
ABC-SVM & 0.4924 & $85.5 \%$ & 5.5706 & 23.6695 & $9.085092 \mathrm{~s}$ \\
\hline
\end{tabular}

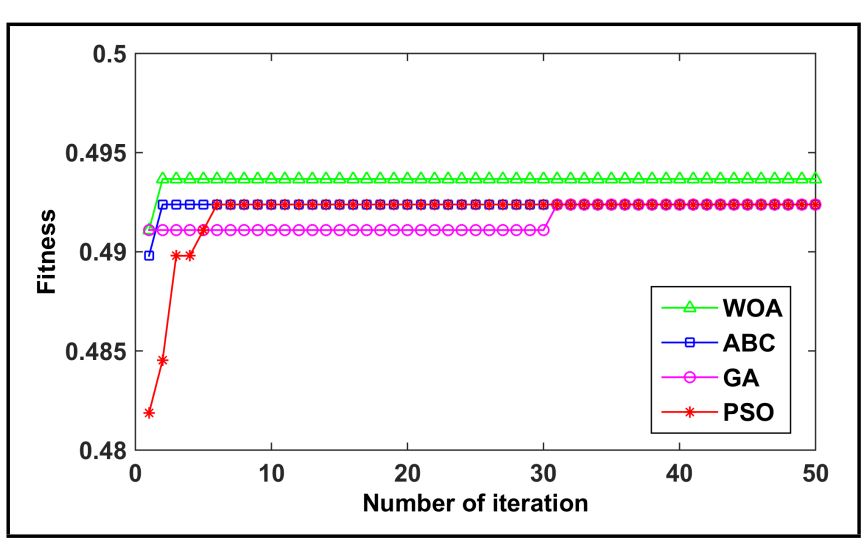

Figure 17. Iterative process of several optimization methods.

Table 6. The recognition rate of different classification methods.

\begin{tabular}{|c|c|c|c|}
\hline Method & $\begin{array}{c}\text { Number of correct } \\
\text { identification samples }\end{array}$ & $\begin{array}{c}\text { Number of } \\
\text { test samples }\end{array}$ & $\begin{array}{c}\text { Recognition } \\
\text { rate }\end{array}$ \\
\hline RCMFE-ISVM & 199 & 200 & $99.5 \%$ \\
RCMFE-NBN & 193 & 200 & $96.5 \%$ \\
RCMFE-KNN & 183 & 200 & $91.5 \%$ \\
RCMFE-ANN & 193 & 200 & $96.5 \%$ \\
\hline
\end{tabular}

ward trend, and the recognition rate was always higher than other methods. It demonstrates that the ISVM proposed in this paper was more robust to training samples than other methods. The ISVM can still maintain a high recognition rate in the case of small samples, which was more suitable for practical application.

Similarly, the influence of different scale factors on diagnostic recognition rates was analyzed in this section. As shown in Fig. 20, when the scale factor was greater than or equal to 3 , the fault recognition rate reached $98 \%$ and above. It was shown that when the scale factor was greater than 3 , the fault recognition rate tended to be stable. If the scale factor was too large, the amount of calculation increased. Therefore, the analysis results were consistent with Case 1 , and in this case, it was more appropriate to set the scale factor at $3 \sim 6$.

\section{CONCLUSIONS}

In this article, a novel method based on the RCMFE and ISVM is proposed for rolling bearings fault diagnosis under variable working conditions. To deal with the non-stationary and non-linear characteristics of bearing fault vibration signal, the RCMFE is employed to provide representative features. Further, the ISVM based on the WOA is proposed to identify the fault pattern of rolling bearing. The WOA is used to optimize the regularization parameter and the RBF kernel parameter of the SVM, which can affect the classification efficiency of the SVM. After verification of the proposed method using bearing fault data of the MFPT and Case Western Reserve University, the following conclusions are drawn.

1. The proposed method is a simple and effective tool for the fault diagnosis of rolling bearings in variable working conditions. It has the advantages of a simple operation process, less historical data, and no manual operation. It can detect the fault of rolling bearing variable working conditions automatically. It shows that the proposed method is more versatile. These characteristics show that the proposed method is more suitable for engineering practice.

2. The RCMFE is applied to extract bearing fault features under variable operating conditions. The experimental data prove that the RCMFE can effectively reflect the bearing fault characteristics under variable working conditions. This feature extraction method is simple and does not need other processing such as dimension reduction, which is conducive to application in engineering practice. The performance of the RCMFE in bearing fault diagnosis is more competitive than other nonlinear dynamic analysis methods such as the MFE and MSE. The 


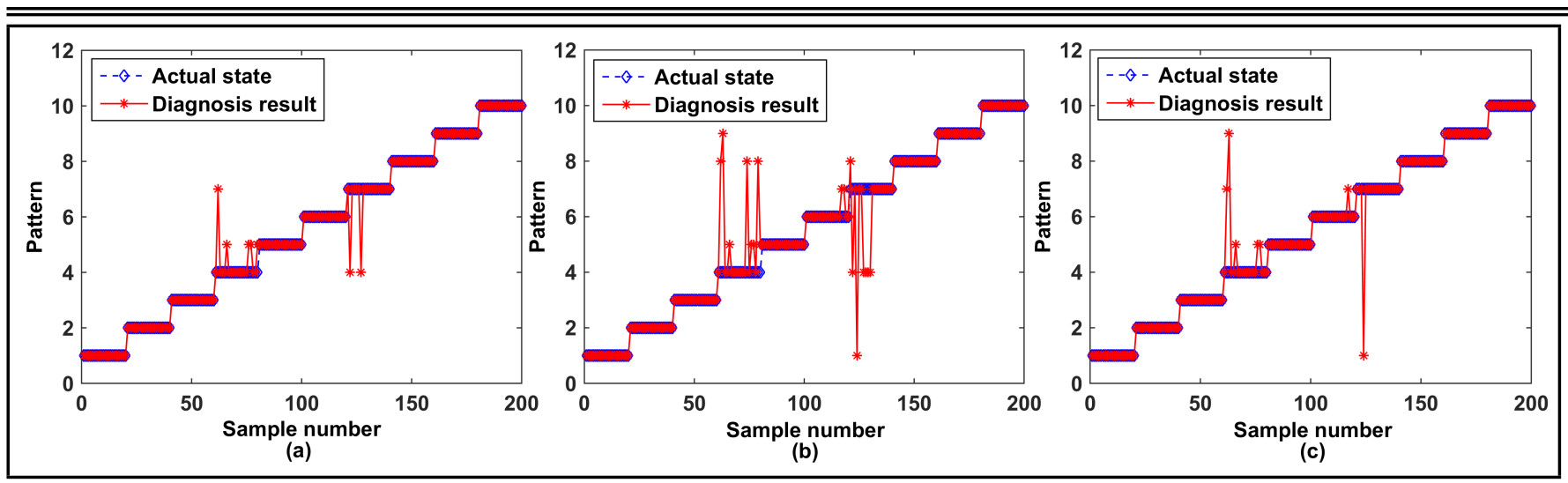

Figure 18. Diagnosis results of different classifiers: (a) RCMFE-NBN, (b) RCMFE-KNN, (c) RCMFE-ANN.

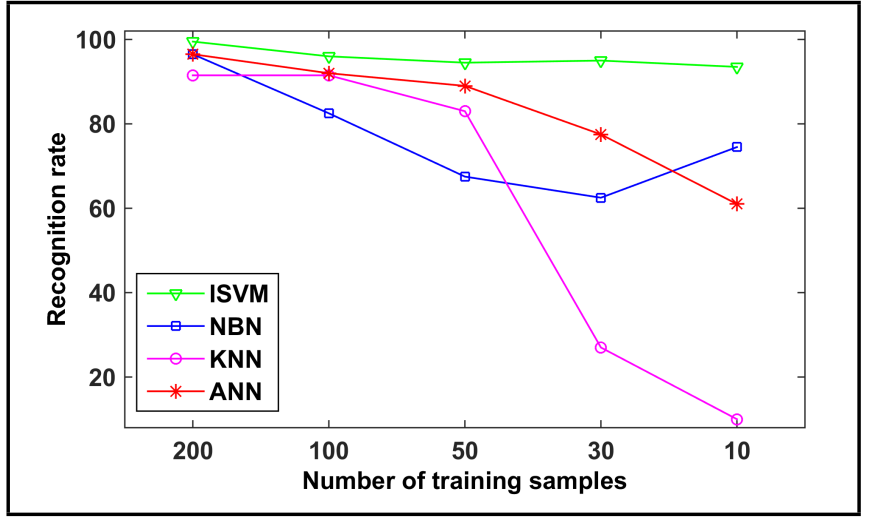

Figure 19. Fault recognition rate of different training samples.

influence of parameter setting on the application of the RCMFE in bearing fault diagnosis is analyzed, which has reference significance for the practical application of engineering.

3. The experimental results show that the ISVM proposed in this paper is an effective classifier for bearing fault diagnosis under variable working conditions. The ISVM has proved to be more effective than traditional classification methods and requires less training samples. Moreover, the WOA in the ISVM has a better optimization effect and a faster convergence speed than traditional optimization algorithms such as the GA, PSO and ABC.

\section{REFERENCES}

1 Djurdjanovic, D., Lee, J., and Ni, J. Watchdog Agent-an infotronics-based prognostics approach for product performance degradation assessment and prediction, Advanced Engineering Informatics, 17 (3-4), 109-125, (2003). https://dx.doi.org/10.1016/j.aei.2004.07.005

2 Gelman, L., Murray, B., Patel, T. H., and Thomson, A. Vibration diagnostics of rolling bearings by novel nonlinear non-stationary wavelet bicoherence technology, Engineering Structures, 80 (1), 514-520, (2014). https://dx.doi.org/10.1016/j.engstruct.2014.08.030

3 Wang, T., Liang, M., Li, J., and Cheng, W. Rolling element bearing fault diagnosis via fault characteristic order (FCO) analysis, Mechanical Systems and Signal Processing, 45 (1), 139-153, (2014). https://dx.doi.org/10.1016/j.ymssp.2013.11.011

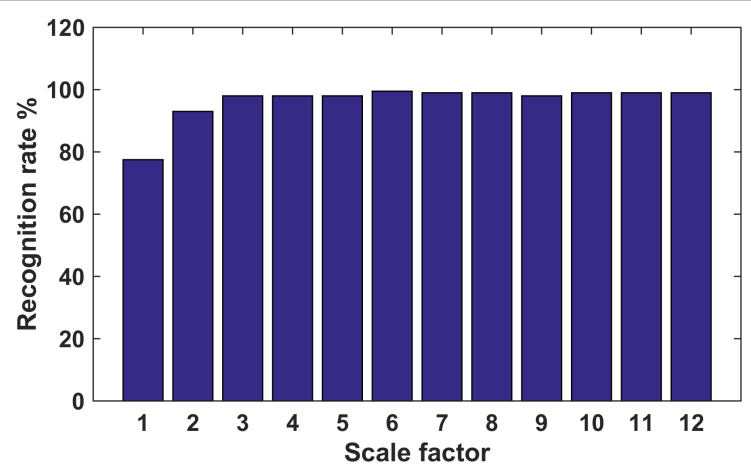

Figure 20. Fault recognition rate of different scale factor.

4 Jena, D. P. and Panigrahi, S. N. Automatic gear and bearing fault localization using vibration and acoustic signals, Applied Acoustics, 98, 20-33, (2015). https://dx.doi.org/10.1016/j.apacoust.2015.04.016

5 Tandon, N. and Choudhury, A. A review of vibration and acoustic measurement methods for the detection of defects in rolling element bearings, Tribology International, 32 (8), 469-480, (1999). https://dx.doi.org/10.1016/S0301679X(99)00077-8

6 Glowacz, A., Glowacz, W., Glowacz, Z., and Kozik, J. Early fault diagnosis of bearing and stator faults of the single-phase induction motor using acoustic signals, Measurement, 113, 1-9, (2018). https://dx.doi.org/10.1016/j.measurement.2017.08.036

7 Peng, Z. and Kessissoglou, N. An integrated approach to fault diagnosis of machinery using wear debris and vibration analysis, Wear, 255 (7-12), 1221-1232, (2003). https://dx.doi.org/10.1016/S0043-1648(03)00098-X

${ }^{8}$ Loutas, T. H., Roulias, D., Pauly, E., and Kostopoulos, V. The combined use of vibration, acoustic emission and oil debris on-line monitoring towards a more effective condition monitoring of rotating machinery, Mechanical Systems and Signal Processing, 25 (4), 1339-1352, (2011). https://dx.doi.org/10.1016/j.ymssp.2010.11.007

9 Janssens, O., Schulz, R., Slavkovikj, V., Stockman, K., Loccufier, M., Van de Walle, R., and Van Hoecke, S. Thermal image based fault diagnosis for rotating machinery, Infrared Physics \& Technology, 73, 78-87, (2015). https://dx.doi.org/10.1016/j.infrared.2015.09.004 
10 Younus, A. M. D. and Yang, B. S. Intelligent fault diagnosis of rotating machinery using infrared thermal image, $E x$ pert Systems with Applications, 39 (2), 2082-2091, (2012). https://dx.doi.org/10.1016/j.eswa.2011.08.004

11 Tran, V. T., Yang, B. S., Gu, F., and Ball, A. Thernal image enhancement using bi-dimensional empirical mode decomposition in combination with relevance vector machine for rotating machinery fault diagnosis, Mechanical Systems and Signal Processing, 38 (2), 601-614, (2013). https://dx.doi.org/10.1016/j.ymssp.2013.02.001

12 Dron, J. P., Rasolofondraibe, L., Bolaers, F., and Pavan, A. High-resolution methods in vibratory analysis: application to ball bearing monitoring and production machine, International Journal of Solids and Structures, 38 (2425), 4293-4313, (2001). https://dx.doi.org/10.1016/S00207683(00)00277-8

13 Altmann, J. and Mathew, J. Multiple band-pass autoregressive demodulation for rolling element bearing fault diagnosis, Mechanical Systems and Signal Processing, 15 (5), 963-977, (2001). https://dx.doi.org/10.1006/mssp.2001.1410

14 Antoni, J. The spectral kurtosis: a useful tool for characterising non-stationary signals, Mechanical Systems and Signal Processing, 20 (2), 282-307, (2006). https://dx.doi.org/10.1016/j.ymssp.2004.09.001

15 Antoni, J. and Randall, R. B. The spectral kurtosis: application to the vibratory surveillance and diagnostics of rotating machines, Mechanical Systems and Signal Processing, 20 (2), 308-331, (2006). https://dx.doi.org/10.1016/j.ymssp.2004.09.002

16 Wang, Y. and Liang, M. An adaptive SK technique and its application for fault detection of rolling element bearings, Mechanical Systems and Signal Processing, 25 (5), 1750-1764, (2011). https://dx.doi.org/10.1016/j.ymssp.2010.12.008

17 Wang, D., Tse, P. W., and Tsui, K. L. An enhanced kurtogram method for fault diagnosis of rolling element bearing, Mechanical Systems and Signal Processing, 35 (1-2), 176-199, (2013). https://dx.doi.org/10.1016/j.ymssp.2012.10.003

18 Singh, J., Darpe, A. K., and Singh, S. P. Rolling element bearing fault diagnosis based on overcomplete rational dilation wavelet transform and auto-correlation of analytic energy operator, Mechanical Systems and Signal Processing, 100, 662-693, (2018). https://dx.doi.org/10.1016/j.ymssp.2017.06.040

19 Wang, D., Zhao, Y., Yi, C., Tsui, K., and Lin, J. Sparsity guided empirical wavelet transform for fault diagnosis of rolling element bearings, Mechanical Systems and Signal Processing, 101, 292-308, (2018). https://dx.doi.org/10.1016/j.ymssp.2017.08.038

20 Guo, Y., Liu, T., Na, J., and Fung, R. Envelope order tracking for fault detection in rolling element bearings, Journal of Sound and Vibration, 331 (25), 5644-5654, (2012). https://dx.doi.org/10.1016/j.jsv.2012.07.026
21 Wang, T., Liang, M., Li, J., Cheng, W., and Li, C. Bearing fault diagnosis under unkown variable speed via gear noise cancellation and rotational order sideband identification, Mechanical Systems and Signal Processing, 62-63, 30-53, (2015). https://dx.doi.org/10.1016/j.ymssp.2015.03.005

22 Guo, W., Tse, P. W., and Djordjevich, A. Faulty bearing signal recovery from large noise using a hybrid method based on spectral kurtosis and ensemble empirical mode decomposition, Measurement, 45 (5), 1308-1322, (2012). https://dx.doi.org/10.1016/j.measurement.2012.01.001

23 Xue, X., Zhou, J., Xu, Y., Zhu, W., and Li, C. An adaptively fast ensemble empirical mode decomposition method and its applications to rolling element bearing fault diagnosis, Mechanical Systems and Signal Processing, 62-63, 444-459, (2015). https://dx.doi.org/10.1016/j.ymssp.2015.03.002

24 Liao, L. Discovering prognostic features using genetic programming in remaining useful life prediction, IEEE Transactions on Industrial Electronics, 61 (5), 2464-2472, (2014). https://dx.doi.org/10.1109/TIE.2013.2270212

25 Kankar, P. K., Sharma, S. C., and Harsha, S. P. Rolling element bearing fault diagnosis using wavelet transform, Neurocomputing, 74 (10), 1638-1645, (2011). https://dx.doi.org/10.1016/j.neucom.2011.01.021

26 Dong, H., Qi, K., Chen, X., Zi, Y., He, Z., and Li, B. Sifting process of EMD and its application in rolling element bearing fault diagnosis, Journal of Mechanical Science and Technology, 23 (8), 2000-2007, (2009). https://dx.doi.org/10.1007/s12206-009-0438-9

27 Tian, Y., Ma, J., Lu, C., and Wang, Z. Rolling bearing fault diagnosis under variable conditions using LMD-SVD and extreme learning machine, Mechanism and Machine Theory, 90, 175-186, (2015). https://dx.doi.org/10.1016/j.mechmachtheory.2015.03.014

28 Wang, H., Chen, J., and Dong, G. Feature extraction of rolling bearing's early weak fault based on EEMD and tunable Q-factor wavelet transform, Mechanical Systems and Signal Processing, 48 (1-2), 103-119, (2014). https://dx.doi.org/10.1016/j.ymssp.2014.04.006

29 Zosso, D. and Dragomiretskiy, K. Variational Mode Decomposition, IEEE Transactions on Signal Processing, 62 (3), 531-544, (2014). https://dx.doi.org/10.1109/TSP.2013.2288675

${ }^{30} \mathrm{Li}$, Z., Chen, J., Zi, Y., and Pan, J. Independenceoriented VMD to identify fault feature for wheel set bearing fault diagnosis of high speed locomotive, Mechanical Systems and Signal Processing, 85, 512-529, (2017). https://dx.doi.org/10.1016/j.ymssp.2016.08.042

$31 \mathrm{Hu}$, A., Yan, X., and Xiang, L. A new wind turbine fault diagnosis method based on ensemble intrinsic time-scale decomposition and WPT-fractal dimension, Renewable Energy, 83, 767-778, (2015). https://dx.doi.org/10.1016/j.renene.2015.04.063 
${ }^{32}$ Zhang, X., Zhao, C., Dong, H., Liu, X., and Zeng, L. Rolling bearing fault diagnosis using sample entropy and 1.5 dimension spectrum based on EMD, Applied Mechanics and Materials, 278-280, 1027-1031, (2013). https://dx.doi.org/10.4028/www.scientific.net/AMM.278280.1027

${ }^{33}$ Zheng, J., Cheng, J., and Yang, Y. A rolling bearing fault diagnosis approach based on LCD and fuzzy entropy, Mechanism and Machine Theory, 70, 441-453, (2013). https://dx.doi.org/10.1016/j.mechmachtheory.2013.08.014

${ }^{34}$ Zhang, L., Xiong, G., Liu, H., Zou, H., and Guo, W. Bearing fault diagnosis using multi-scale entropy and adaptive neuro-fuzzy inference, Expert Systems with Applications, 37 (8), 6077-6085, (2010). https://dx.doi.org/10.1016/j.eswa.2010.02.118

35 Zheng, J., Cheng, J., Yang, Y., and Luo, S. A rolling bearing fault diagnosis method based on multi-scale fuzzy entropy and variable predictive model-based class discrimination, Mechanism and Machine Theory, 78, 187-200, (2014). https://dx.doi.org/10.1016/j.mechmachtheory.2014.03.014

${ }^{36} \mathrm{Li}$, Y., Wang, X., Si, S., and Huang, S. Entropy based fault classification using the Case Western Reserve University data: A benchmark study, IEEE Transactions on Reliability, 69 (2), 754-767, (2020). https://dx.doi.org/10.1109/TR.2019.2896240

${ }^{37} \mathrm{Li}$, Y., Wang, X., Liu, Z., Liang, X., and Si, S. The entropy algorithm and its variants in the fault diagnosis of rotating machinery: a review, IEEE Access, 6, 66723-66741, (2018). https://dx.doi.org/10.1109/access.2018.2873782

38 Azami, H., Fernandez, A., and Escudero, J. Refined multiscale fuzzy entropy based on standard deviation for biomedical signal analysis, Medical \& Biological Engineering \& Computing, 55 (11), 2037-2052, (2017). https://dx.doi.org/10.1007/s11517-017-1647-5

39 Zheng, J., Jiang, Z., and Pan, H. Sigmoid-based refined composite multiscale fuzzy entropy and $t$ SNE based fault diagnosis approach for rolling bearing, Measurement, 129, 332-342, (2018). https://dx.doi.org/10.1016/j.measurement.2018.07.045

40 Li, Y., Miao, B., Zhang, W., and Chen, P. et al. Refined composite multiscale fuzzy entropy: Localized defect detection of rolling element bearing, Journal of Mechanical Science and Technology, 33 (1), 109-120, (2019). https://dx.doi.org/10.1007/s12206-018-1211-8

${ }^{41}$ Zhang, N., Wu, L., Yang, J., and Guan, Y. Naïve bayes bearing fault diagnosis based on enhanced independence of data, Sensor, 18 (2), 1-17, (2018). https://dx.doi.org/10.3390/s18020463

42 Dong, S., Xu, X., and Chen, R. Application of fuzzy C-means method and classification model of optimized K-nearest neighbor for fault diagnosis of bearing, Journal of the Brazilian Society of Mechanical Science and Engineering, 38 (8), 2255-2263, (2016). https://dx.doi.org/10.1007/s40430-015-0455-9
43 Ali, J., Fnaiech, N., Saidi, L., Chebel-Morello, B., and Fnaiech, F. Application of empirical mode decomposition and artificial neural network for automatic bearing fault diagnosis based on vibration signals, Applied Acoustics, 89, 16-27, (2015). https://dx.doi.org/10.1016/j.apacoust.2014.08.016

${ }^{44}$ Su, Z., Tang, B., Liu, Z., and Qin, Y. Multi-fault diagnosis for rotating machinery based on orthogonal supervised linear local tangent space alignment and least square support vector machine, Neurocomputing, 157, 208-222, (2015). https://dx.doi.org/10.1016/j.neucom.2015.01.016

45 Yang, Y., Yu, D., and Cheng, J. A fault diagnosis approach for roller bearing based on IMF envelope spectrum and SVM, Measurement, 40 (9-10), 943-950, (2007). https://dx.doi.org/10.1016/j.measurement.2006.10.010

${ }^{46}$ Li, Y., Yang, Y., Wang, X., Liu, B., and Liang. X. Early fault diagnosis of rolling bearings based on hierarchical symbol dynamic entropy and binary tree support vector machine, Journal of Sound and Vibration, 428, 72-86, (2018). https://dx.doi.org/10.1016/j.jsv.2018.04.036

47 Samanta, B. Gear fault detection using artificial neural networks and support vector machines with genetic algorithms, Mechanical Systems and Signal Processing, 18 (3), 625-644, (2004). https://dx.doi.org/10.1016/S08883270(03)00020-7

48 Bordoloi, D. J. and Tiwari, R. Optimal multifault classification of gears with integration of evolutionary and SVM algorithms, Mechanism and Machine Theory, 73, 49-60, (2014). https://dx.doi.org/10.1016/j.mechmachtheory.2013.10.006

${ }^{49}$ Potts, J. C., Giddens D., and Yadav, B. The development and evaluation of an improved genetic algorithm based on migration and artificial selection, IEEE Transactions on Systems, Man, and Cybernetics, 24 (1), 73-86, (1994). https://dx.doi.org/10.1109/21.259687

${ }^{50}$ Shi, Y. H. and Eberhart, R. A modified particle swarm optimizer, Proceedings of the IEEE International Conference on Evolutionary Computation, Anchorage, (1998). https://dx.doi.org/10.1109/ICEC.1998.699146

${ }^{51}$ Karaboga, D. and Akay, B. A comparative study of artificial bee colony algorithm, Applied Mathematics and Computation, 214 (1), 108-132, (2009). https://dx.doi.org/10.1016/j.amc.2009.03.090

52 Mirjalili, S. and Lewis, A. The whale optimization algorithm, Advances in Engineering Software, 95, 51-67, (2016). https://dx.doi.org/10.1016/j.advengsoft.2016.01.008

53 Costa, M., Goldberger, A. L., and Peng, C. K. Multiscale entropy analysis of biological signals, Physical Review E, 71 (2), 021906, (2005). https://dx.doi.org/10.1103/PhysRevE.71.021906 